\section{Factores de riesgo del comportamiento y preparación de aficionados inscritos a carreras atléticas de fondo de 18-64 años en Bogotá, Colombia, 2014}

\author{
Behavioral risk factors and readiness in amateur \\ marathon runners 18-64 years of age in Bogotá, \\ Colombia, 2014
}

Fatores de risco comportamentais e prontidão em corredores amadores de 18 a 64 anos, inscritos em corridas de fundo em Bogotá, Colômbia, 2014

\begin{abstract}
Resumen
La participación en carreras atléticas de calle ha aumentado; esto requiere detectar riesgos previos al esfuerzo físico. El objetivo fue identificar factores de riesgo del comportamiento y preparación física de inscritos a una carrera. Estudio transversal en aficionados de 18-64 años. Encuesta digital con módulos de IPAQ, Par-Q+ y STEPwise. Muestreo aleatorio sistemático con $n=510$, para una inactividad física esperada de $35 \%$ ( $\pm 5 \%$ ). Se evaluó nivel de actividad física, consumo de alcohol (peligroso), de fruta, verdura, tabaco y sal, y preparación. El cumplimiento de actividad física fue 97,4\%; $2,4 \%$ consume un nivel óptimo de fruta o verdura (diferencias por edad, sexo y estrato), 3,7\% fuma y 44,1\% consumo peligroso de alcohol. El 19,8\% reportó Par-Q+ positivo y 5,7\% requiere supervisión. Hay diferencias por trabajo y estudio. Los aficionados cumplen el nivel de actividad física; pero no de otros factores. Una estrategia de seguridad en el atletismo de calle es evaluar los factores de riesgo relacionados con el estilo de vida, así como la preparación.
\end{abstract}

Carrera; Muerte Súbita; Atletas; Enfermedad Crónica; Factores de Riesgo
María del Pilar Ramírez-Góngora 1,2 Franklyn Edwin Prieto-Alvarado 1

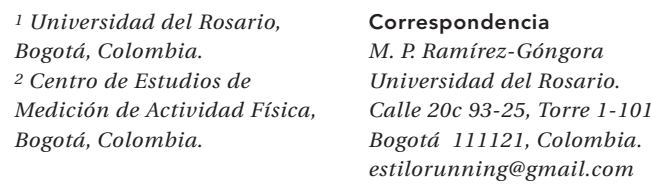

1 Universidad del Rosario, Bogotá, Colombia.

2 Centro de Estudios de Medición de Actividad Física, Bogotá, Colombia. 


\section{Introducción}

Los movimientos mundial y local de carreras atléticas de calle son una opción de práctica de actividad física y ejercicio regular, que ganan aficionados diariamente. Los reportes de participantes que terminaron una maratón en Estados Unidos entre 1990 y 2012, oscilaron entre 1.199.200 y 8.699 .000 mujeres, y entre 3.587 .800 y 6.835 .000 hombres (Running USA. Statistics. http://www.runningusa. org/statistics, accedido el Mar/2014). Así como aumenta el número de participantes, desconocer los posibles factores de riesgo presentes en ellos, desde su preparación física hasta sus comportamientos relacionados con el estilo de vida, incrementa la posibilidad de padecer un evento, en pruebas como la maratón y carreras de fondo, que demandan mayor esfuerzo cardiovascular 1,2,3,4.

Las carreras recreativas han aumentado en Bogotá, Colombia. Según el calendario atlético, para el 2014 fueron programadas 29 carreras, de 40 nacionales 5 . El promedio de carreras, es de 1,8 por mes. Según los reportes de la Media Maratón de Bogotá, una de las carreras más importantes, la participación de aficionados aumentó. En 2002 fueron 20.820 corredores y en 2010, 44.192 ${ }^{6}$. El nivel y la continuidad en la participación en las carreras atléticas por aficionados demandan valorar las condiciones previas de los inscritos, no sólo de los tiempos de carrera, sino de las condiciones de salud y los factores de riesgo del comportamiento, para desarrollar estrategias que mitiguen los riesgos en salud. Esto no se ha realizado en Colombia.

La exposición a esfuerzos físicos de larga duración requiere un nivel de preparación física para disminuir los riesgos de tipo cardiovascular 7,8. La exigencia de las pruebas, la distancia y el nivel requerido de preparación física podrían exponer a eventos cardiovasculares repentinos o muerte súbita 9,10. El ejercicio vigoroso agudiza transitoriamente el riesgo 11,12,13, cuando no se cuenta con una adecuada preparación física o existen antecedentes clínicos, factores de riesgo, en algunos casos desconocidos o no percibidos 3,10,12,14.

Los organizadores de carreras en el mundo como los maratones de Boston, Londres, Berlín, entre otros, realizan un tamizaje de riesgo o evaluaciones previas a los atletas, para detectar y reducir el riesgo cardiovascular 12,15,16. Ante los eventos en las diferentes carreras, Maron reportó por primera vez que la incidencia de muerte súbita variaba entre 0,54 y 2,1 eventos por cada 100.000 participantes, con mayor afectación entre la sexta y octava década .

Según Maron 8 (p. 477), "el screening, preparticipación (sic) con historia y examen físico por sí solos, sin test invasivos, no tiene suficiente poder para garantizar la detección de anormali- dades críticas a nivel poblacional". Sin embargo, "claramente, si los atletas son obesos, fuman, tienen presión arterial alta, lípidos con niveles anormales, o tienen una historia familiar de enfermedad cardiovascular, ellos tendrán aumentado el riesgo" 17 (p. 383).

La implementación de tamizajes poblacionales con exámenes médicos y clínicos es poco factible, por su costo y la dificultad de masificar su aplicación. Los cuestionarios son una alternativa que permite identificar las condiciones previas de los corredores, como los factores del comportamiento que son determinantes de enfermedades crónicas y de riesgos potenciales ante la participación en las carreras, para mejorar las acciones sobre la seguridad del atleta 14,18 .

El objetivo del estudio fue identificar los factores de riesgo del comportamiento y el estado previo de preparación de los corredores aficionados de carreras atléticas de 18 a 64 años en Bogotá.

\section{Materiales y métodos}

Es un estudio transversal, tipo evaluación rápida. Se administró un cuestionario en la fase previa de una carrera atlética de fondo (10 y $21 \mathrm{~km})$, en Bogotá, durante 2014; en el proceso de entrega de kits de carrera a los inscritos entre 18 y 64 años.

El tamaño de muestra se calculó para una población de 30.000 potenciales inscritos, un nivel esperado de inactividad física de $35 \%, 10 \%$ menor a lo reportado en la Encuesta Nacional de Salud y Nutrición (ENSIN) 19, por considerarlos potencialmente más activos, con un error admisible del $4,5 \%$ y una confianza al $95 \%$. Se definió la fracción para un muestreo aleatorio sistemático. Se evaluaron a 510 personas, considerando un $20 \%$ adicional por "no participación". El tamaño de muestra, establece como estimación principal, la inactividad física, sin embargo, permite obtener los otros indicadores clave como preparación y los factores del comportamiento explorados, con un error esperado menor del $5 \%$.

Los criterios de inclusión fueron: hombres y mujeres corredores aficionados entre 18 y 64 años, inscritos en una carrera atlética de fondo en Bogotá. Los criterios de exclusión fueron: tener alguna condición de discapacidad, ser atleta élite, reporte de un tiempo menor a 45 minutos, en los $10 \mathrm{~km}$.

El cuestionario estuvo compuesto por módulos seleccionados del International Physical Activity Questionnaire (IPAQ) 20,21, Physical Activity Readiness Questionnaire for Everyone (Par-Q+) 2013 22,23,24 y el Método Progresivo de la Organización Mundial de la Salud (OMS) para la vigilancia de los factores de riesgo de las enfermedades crónicas (STEPS) STEPwise 25. 
Del cuestionario STEPwise, se seleccionaron los indicadores principales de factores de riesgo del comportamiento relacionados con: consumo de tabaco, alcohol, frutas y verduras y sal, de acuerdo con los indicadores del Plan Decenal de Salud Pública.

El IPAQ es un cuestionario que ha mostrado validez y confiabilidad, comparado con acelerometría, para medir niveles poblacionales de actividad e inactividad física, entre 18 y 65 años; y se ha recomendado la utilización de los dominios de actividad física en el tiempo libre y por transporte, de la versión larga, para encuestas poblacionales 21, como la ENSIN 19.

El Par-Q+, es avalado por el American College of Sports Medicine 23,26,27. Warburton 23,24 comparó el Par-Q+ con el Par-Q y demostró una especificidad de 0,99-1 y un coeficiente de correlación de Pearson de 0,99 para valorar el riesgo de enfermedades crónicas y promover el ingreso seguro a la actividad física. Entre sus características se encuentran: la facilidad de aplicación en personas físicamente activas, ser aplicado por profesionales especializados del campo del ejercicio y poder referir los casos positivos de riesgo a un médico 28.

El cuestionario final, incluyó los siguientes módulos:

a) Módulo A: indagó sobre variables sociodemográficas. Se establecieron grupos de edad, de 18 a 29, de 30 a 44 y de 45 a 64 años; dos grupos de estrato socioeconómico: bajo-medio $(1,2$ y 3$)$ y medio-alto (4,5 y 6), y para el nivel educativo, un grupo entre básica primaria y nivel técnico y otro que incluyó pregrado y postgrado. La ocupación durante los últimos 30 días se agrupó como: trabajo y estudio, buscar trabajo, ser pensionado y dedicarse a las tareas del hogar.

b) Módulo B: con preguntas relacionadas con hábitos de vida, seleccionadas del instrumento STEPwise de OMS 25. Consumo peligroso de alcohol en hombres mayor o igual a 5 tragos y para mujeres mayor o igual a 4 tragos, en al menos una ocasión durante los últimos 30 días. Consumo actual de tabaco y consumo de sal adicional se categorizó de forma dicotómica 29,30. El consumo de frutas y verduras se clasificó según el cumplimiento de consumo semanal recomendado por la OMS, de cinco porciones diarias entre frutas y verduras, estableciendo consumo muy bajo al menor a 15 porciones/semana; bajo, entre 15 y 24; sub-óptimo, 25 y 34 y óptimo, mayor de 35 porciones 31 .

c) Módulo C: se seleccionaron los dominios del IPAQ versión larga, para nivel de actividad física moderada y vigorosa en tiempo libre y de transporte, valorada con un nivel de cumplimiento mayor o igual a 150 minutos por semana de actividad moderada y mayor o igual a 75 minutos de actividad vigorosa por semana en cada uno de los niveles y total 32 .

d) Módulo D: se administró todo el Par-Q+ que incluye preguntas relacionadas con el riesgo cardiovascular y enfermedades crónicas valoradas con escalas dicotómicas.

Antes de la administración en campo se probó el instrumento en una carrera previa de 2014 y a partir de los hallazgos se ajustó. El procedimiento en los dispositivos móviles fue probado en dos momentos: en un aula de clase con 20 estudiantes y con los encuestadores para revisar dudas, tanto del cuestionario, como de la operación. La concordancia en las preguntas clave fue mayor de 0,80 .

Las encuestas fueron realizadas cara a cara, a partir de la selección aleatoria de los individuos en la fila de reclamo de los kit de carrera, utilizando como fracción de muestreo 17. La administración de las encuestas se dividió en los tres días de entrega de kit, logrando porciones iguales de muestra durante la jornada de 8:00 a 6:00 pm. El tiempo para diligenciar el cuestionario fue entre $10 \mathrm{y}$ 12 minutos.

Cada participante fue informado sobre el objetivo del estudio y se le invitó a responder las preguntas, y se amplió la información para garantizar el proceso de comprensión y consentimiento o no. El cuestionario fue diligenciado en tabletas digitales que operaron un formulario elaborado en Google Forms (https://www.google.com/forms/ about/). A medida que se encuestaba, automáticamente se cargaba a la base de datos. Un ingeniero de sistemas verificó el funcionamiento del proceso y se realizaron cortes cada hora para producir copias de seguridad.

Se realizó análisis univariado, determinando para variables cualitativas, la distribución de frecuencias absolutas y relativas porcentuales. Las variables cuantitativas se analizaron en sus medidas de tendencia central como el promedio y las medidas de dispersión, como la desviación estándar, con su intervalo al 95\% de confianza (IC95\%). Para estratificar el análisis se establecieron agrupaciones de categorías de las diferentes variables.

Se efectúo un análisis bivariado para explorar asociaciones entre las variables sociodemográficas y los factores del comportamiento, cumplimiento del nivel de actividad física y preparación. Se aplicó la prueba Mantel \& Haenszael y en algunos casos de acuerdo al número de valores esperados menores de 5 se aplicó la prueba binomial de Mid.p exacto ${ }^{33}$. El nivel de significancia fue del $5 \%$. Los intervalos de confianza de proporciones simples se calcularon al $95 \%$.

Las personas con al menos una pregunta afirmativa en el Par-Q+ fueron acompañados a un espacio habilitado temporalmente para su 
valoración por un médico especialista en medicina deportiva, quien administró el $e$-Physical Activity Readiness Medical Examination (PARmedX) 24 y explicó los cuidados a tener durante la carrera, así como los riesgos. Se imprimieron folletos informativos con recomendaciones a los participantes. Por correo electrónico se enviará individualmente un folleto en el que se informarán los resultados del estudio y las recomendaciones para aumentar la seguridad mediante la prevención de eventos cardiovasculares. Se contó con una cuenta de correo electrónico del estudio, en la que se recibieron las inquietudes y se enviaron a los participantes que asentían querer conocer el análisis de su reporte.

Se envió un reporte a la organización de la carrera sobre los hallazgos; en una reunión para compartir detalles y evidenciar la importancia del tamizaje para valorar el riesgo y establecer estrategias de mayor seguridad para el participante y el organizador.

\section{Resultados}

La muestra final fue de 506 participantes. La edad promedio fue de 39,5 $\pm 11,16$ años. La participación de hombres fue de $70,55 \%$. El 53,56\% pertenece a los estratos bajo-medio; el nivel educativo que predominó fue pregrado y postgrado, con un $83,41 \%$. La mayor ocupación en los últimos 30 días fue trabajar, con el 43,93\%. Para el 33,99\% era la primera carrera en los últimos seis meses. El promedio de carreras fue de 2,34 $\pm 3,34$ (Tabla 1 ).

El 89,53\% cumple el nivel recomendado de actividad física semanal por recreación, deporte, ejercicio o en su tiempo libre. La actividad física moderada constituye la forma predominante con el $69,96 \%$. El cumplimiento de actividad vigorosa es del 44,47\%. El 29,64\% cumple con las recomendaciones de actividad física por transporte entre caminata y bicicleta. El cumplimiento de las recomendaciones es del 97,43\% entre el tiempo libre y para el transporte (Tabla 2). El nivel de cumplimiento de las recomendaciones de nivel de actividad física no reportó diferencias por variables sociodemográficas.

El promedio de consumo de frutas y verduras fue de $12,5 \pm 8,8$ porciones. El $2,4 \%$ de los participantes cumplen con el consumo recomendado de frutas y verduras. El $65 \%$ reporta nunca agregar sal a las comidas antes de probarlas. El 3,69\% fuma y el $49,6 \%$ ha consumido alcohol en los últimos 30 días. Con referencia al consumo peligroso de alcohol, los hombres presentan el 11,8\% a diferencia de las mujeres, con 4,54\%. El promedio de mayor número de tragos consumidos fue de 1,98 $\pm 3,92$. Se encontraron diferencias en el consumo de tabaco por estrato (valor de $\mathrm{p}=0,03$ ) y en el consumo de alcohol por grupos de edad (valor de $\mathrm{p}<0,05$ ) Frente al consumo recomendado de frutas y verduras se observaron diferencias por sexo (valor de $\mathrm{p}=0$,028) (Tabla 3).

Según el Par-Q+ (preparación), el 19,76\% de los inscritos reportó una condición positiva que amerita una valoración médica previa. El 3,56\% aduce tener una condición cardiovascular. El 5,73\% refiere tener que realizar actividad física supervisada. El 7,71\% está medicado por tener una condición crónica (Tabla 4). No se presentaron diferencias significativas por sexo, edad, estrato. Hay asociación significativas con estudiar y trabajar (valor de $\mathrm{p}<0,01)$.

\section{Discusión}

Según estadísticas nacionales reportadas en el repositorio de datos Finalap (http://www.fina lap.com, accedido el Nov/2014) la proporción de hombres y mujeres que participan en las carreras es de $67,8 \%$ y $32,13 \%$, respectivamente, que coincide con las del estudio. El promedio de edad encontrado es similar al estudio realizado por Donado et al. ${ }^{34}$. Los participantes seleccionados en este estudio son representativos, tanto de la carrera seleccionada, como de las carreras a nivel nacional.

Varios estudios han reportado 1,2,7,35 los posibles riesgos cardiovasculares de los corredores recreativos, que pasan desapercibidos o se presentan súbitamente, a menos que se realicen estudios que detecten enfermedades crónicas 1 o factores de riesgo del comportamiento 1,36,37. La administración de cuestionarios es un método costo-efectivo, que genera información sobre estos antecedentes, previos a la exposición a un mayor esfuerzo físico, que afectarían la salud antes, durante e incluso, después de la carrera.

Se encontró que el 19,76\% reportó una condición que debería tener una revisión médica previa. El 5,93\% refirió una causa cardiovascular o hipertensión 26,38,39,40,41. Esto evidencia la necesidad de profundizar en las condiciones individuales de seguridad frente al riesgo, teniendo en cuenta que la exposición al esfuerzo físico y cardiovascular, como el que demanda cubrir una distancia de $10 \mathrm{o}$ más kilómetros, puede detonar en un infarto o en un evento cardiovascular, cuando no se conocen o no hay un seguimiento de los valores de presión arterial, lípidos en sangre y factores de riesgo 18,39.

La implementación del cuestionario previa a la participación en eventos atléticos del ámbito local disminuye el desconocimiento de las características particulares de los corredores aficionados. En una carrera de características similares se encontró una prevalencia de riesgo por Par-Q+ de 14,9\% para mujeres y $13,5 \%$ para hombres 34 . Stafford 
Características sociodemográficas y experiencia atlética de los inscritos a una carrera de fondo. Bogotá, Colombia, 2014.

\begin{tabular}{|c|c|c|c|}
\hline Variables sociodemográficas & $\mathrm{n}$ & $\%$ & IC95\% \\
\hline \multicolumn{4}{|l|}{ Edad (años) } \\
\hline $18-29$ & 108 & 21,34 & $17,94-25,08$ \\
\hline $30-44$ & 228 & 45,04 & $40,76-49,42$ \\
\hline $45-64$ & 170 & 33,60 & $29,58-37,80$ \\
\hline Hombres & 357 & 70,55 & $66,47-74,40$ \\
\hline Estrato bajo/medio & 271 & 53,56 & $49,20-57,88$ \\
\hline \multicolumn{4}{|l|}{ Ocupación en los últimos 30 días } \\
\hline Trabajar & 358 & 70,75 & $66,67-74,59$ \\
\hline Trabajar y estudiar & 88 & 17,39 & $14,27-20,88$ \\
\hline Estudiar & 12 & 2,37 & $1,29-3,90$ \\
\hline \multicolumn{4}{|l|}{ Nivel educativo } \\
\hline Básico/Medio/Técnico/Otro & 135 & 26,68 & $22,96-30,66$ \\
\hline Pregrado & 176 & 34,78 & $30,72-39,01$ \\
\hline Postgrado & 195 & 38,54 & $34,37-42,84$ \\
\hline \multicolumn{4}{|l|}{ Experiencia atlética } \\
\hline Primera carrera & 74 & 14,62 & $11,75-17,91$ \\
\hline \multicolumn{4}{|c|}{ Número de carreras los últimos seis meses } \\
\hline Ninguna & 172 & 33,99 & $29,96-38,21$ \\
\hline $1-5$ & 289 & 57,11 & $52,77-61,38$ \\
\hline 60 más & 45 & 8,89 & $6,64-11,62$ \\
\hline \multicolumn{4}{|c|}{ Tiempo promedio gasto (distancia: 10km) } \\
\hline Más de 44 minutos & 467 & 89,98 & $89,72-94,38$ \\
\hline No conoce el tiempo & 39 & 10,02 & $5,61-10,28$ \\
\hline
\end{tabular}

IC95\%: intervalo al 95\% de confianza.

et al. 36 evidenciaron que el reconocimiento previo de los riesgos por los corredores aficionados con factores de riesgo coronario y un acercamiento con el médico aumentaría la seguridad en la práctica. Los resultados demuestran que la revisión previa de morbilidad sentida es una tarea necesaria en la antesala de eventos deportivos 7,26,35,41.

El cumplimiento de las recomendaciones de actividad física, de los corredores inscritos fue superior al supuesto de cumplimiento del $65 \% 19$. Se encontró un cumplimiento del 97,43\%, la mayoría en el tiempo libre. La alta prevalencia del nivel recomendado de actividad física en el tiempo libre podría corresponder a las actividades inminentes de preparación para la competencia, teniendo en cuenta el esfuerzo y el objetivo, lo que indicaría que cumplían con los niveles mínimos requeridos de actividad física, en la semana anterior a la competición 32 .

El 44,47\% cumplía con el nivel de actividad física vigorosa, que podría relacionarse con un estado físico apropiado para el esfuerzo a desarrollar en la carrera, que se clasificaría de una intensidad vi- gorosa 4. A futuro, es necesario determinar si esos niveles de cumplimiento son equiparables con un nivel de condición física óptimo o condición previa a la preparación de la carrera.

La ENSIN 2010 reportó un predominio de la actividad física por transporte, con el 39,4\% 6 . En nuestro estudio, la práctica de actividad física en tiempo libre fue del $89,53 \%$ y por transporte del $29,64 \%$. Esto sugeriría que el mantenimiento del nivel de actividad física es temporal y relacionado con la meta de carrera.

Es pertinente afinar si la práctica de las carreras de alguna forma, se convierte en estrategia para ser más activos y, por ende, más saludables. Comparando con el estudio de Donado et al. 34 (p. 345), en dónde "los niveles de no cumplimiento de los 150 minutos recomendados de actividad física fueron del 7,1\% y 12,2\% para hombres y mujeres respectivamente", en nuestro estudio los niveles fueron superiores.

El 2,4\% de los inscritos cumplía con el consumo de frutas o verduras propuesto por la OMS. Comparando los resultados con el estudio de 
Prevalencia de factores de riesgo del comportamiento: consumo de frutas, verduras, sal, tabaco y alcohol, y nivel de actividad física en tiempo libre y transporte de los inscritos a una carrera de fondo. Bogotá, Colombia, 2014.

\begin{tabular}{|c|c|c|c|}
\hline Factores de riesgo del comportamiento & $\mathbf{n}$ & $\%$ & IC95\% \\
\hline Consumo óptimo de frutas y verduras & 12 & 2,40 & $1,29-3,99$ \\
\hline Consumo de sal adicional & 104 & 11,40 & $17,20-24,24$ \\
\hline Consumo de sal adicional siempre & 20 & 3,98 & $2,49-5,93$ \\
\hline Prevalencia de consumo de tabaco (vida) & 36 & 3,69 & $5,10-9,61$ \\
\hline Prevalencia de consumo de tabaco (día) & 10 & 1,97 & $1,00-3,49$ \\
\hline Consumo de alcohol en los últimos 30 días & 251 & 49,60 & $45,26-53,96$ \\
\hline \multicolumn{4}{|l|}{ Frecuencia de consumo de alcohol semanal (días) } \\
\hline 0 & 388 & 76,68 & $72,80-80,15$ \\
\hline $1-3$ & 112 & 22,13 & $18,73-25,95$ \\
\hline $4-7$ & 6 & 1,18 & $0,54-2,56$ \\
\hline \multicolumn{4}{|l|}{ Categoría: consumo de tragos de alcohol } \\
\hline Hombres: mayor a 5 & 60 & 11,80 & $9,30-14,97$ \\
\hline Mujeres: mayor a 4 & 23 & 4,54 & $3,04-6,72$ \\
\hline \multicolumn{4}{|l|}{ Nivel de cumplimiento de actividad física } \\
\hline Caminata & 203 & 40,12 & $35,91-44,44$ \\
\hline Actividad física moderada & 354 & 69,96 & $65,86-73,84$ \\
\hline Actividad física vigorosa & 225 & 44,47 & $40,17-48,82$ \\
\hline Cumplimiento total de actividad física (tiempo libre) & 453 & 89,53 & $86,63-91,97$ \\
\hline \multicolumn{4}{|l|}{ Nivel de actividad física por transporte } \\
\hline Camina & 104 & 20,55 & $17,20-24,24$ \\
\hline Bicicleta & 75 & 14,82 & $11,92-18,12$ \\
\hline Cumplimiento total de actividad física por transporte & 150 & 29,64 & $25,79-33,74$ \\
\hline Cumplimiento total de actividad física en tiempo libre y por transporte & 493 & 97,43 & $95,75-98,56$ \\
\hline
\end{tabular}

IC95\%: intervalo al 95\% de confianza.

Bucaramanga (Colombia), los comportamientos son similares, dado que el bajo consumo fue "el factor de riesgo más prevalente" 42 . Los resultados coinciden con la ENSIN 2010, con el 20,5\% y 1,4\%, respectivamente. Es importante desarrollar estrategias que fortalezcan la prevención de enfermedades crónicas, a través del consumo adecuado de frutas y verduras 43,44. En el 2010, la mayor carga de factor de riesgo latente como causa de mortalidad es el factor dietario 45 .

Con relación al consumo adicional siempre de sal, se observó una prevalencia de 11,4\%, que está relacionado con la hipertensión arterial 46,47. La prevalencia de consumo de alcohol en los últimos treinta días fue del $49,6 \%$, que coincide con el estudio de la Fundación para la Educación y Desarrollo Social (FES) 48 . La OMS proyectó un consumo total de 6,2\% para Colombia en el 2012 (World Health Organization. Global health observatory data repository. http://apps.who.int/gho/data/node. main.A867?lang=en, accedido el 14/Feb/2015). Este es un aspecto importante a considerar, teniendo en cuenta que los reportes son elevados y "que el consumo de alcohol no sólo aumenta el riesgo de lesiones, sino también puede exacerbar los riesgos cardiovasculares y hepáticos" 43 (p. 957) factor que en la ejecución de una carrera puede aumentar el riesgo 10 .

El consumo de tabaco mostró una prevalencia de $3,69 \%$, con diferencias por sexo, lo cual es menor al perfil nacional de la OMS que corresponde al $23,8 \%$ y $11,8 \%$, para hombres y mujeres, respectivamente 49 . Los resultados de nuestro estudio son comparables con un menor hábito tabáquico en quienes practican atletismo 50,51, argumento que confirma Donado et al. 34 en el 2011 reportando una prevalencia de tabaco de $8,1 \%$ mujeres y $7,1 \%$ para hombres.

Planteado el panorama de los factores de riesgo del comportamiento, relacionados con el estilo de vida de los corredores, se observa como los participantes cumplen con las recomendaciones de actividad física, pero no se puede asumir que existe un equilibrio en lo referente a los 
Análisis bivariado de cumplimiento de actividad física, consumo de frutas, verduras y sal adicional, tabaco y alcohol vs. variables sociodemográficas de los inscritos a una carrera de fondo. Bogotá, Colombia, 2014.

\begin{tabular}{|c|c|c|c|c|c|c|c|c|c|c|c|c|}
\hline \multirow[t]{2}{*}{ Categorías } & \multicolumn{2}{|c|}{$\begin{array}{l}\text { Cumplimiento } \\
\text { total de las } \\
\text { recomendaciones de } \\
\text { actividad física }\end{array}$} & \multicolumn{2}{|c|}{$\begin{array}{l}\text { Cumplimiento } \\
\text { de actividad } \\
\text { física vigorosa }\end{array}$} & \multicolumn{2}{|c|}{$\begin{array}{l}\text { Consumo de frutas } \\
\text { y verduras nivel } \\
\text { bajo y muy bajo }\end{array}$} & \multicolumn{2}{|c|}{$\begin{array}{c}\text { Consumo adicional } \\
\text { de sal }\end{array}$} & \multicolumn{2}{|c|}{$\begin{array}{l}\text { Consumo de } \\
\text { tabaco }\end{array}$} & \multicolumn{2}{|c|}{$\begin{array}{l}\text { Consumo de } \\
\text { alcohol }\end{array}$} \\
\hline & $\%$ & IC95\% & $\%$ & IC95\% & $\%$ & IC95\% & $\%$ & IC95\% & $\%$ & IC95\% & $\%$ & IC95\% \\
\hline \multicolumn{13}{|l|}{ Sexo } \\
\hline $\begin{array}{l}\text { Masculino (n } \\
=357)\end{array}$ & 96,90 & $94,5-98,34$ & 48,40 & $\begin{array}{l}43,32- \\
53,63 \text { * }\end{array}$ & 94,40 & $\begin{array}{l}91,46- \\
96,39 *\end{array}$ & 21,29 & $\begin{array}{l}17,35- \\
25,84\end{array}$ & 7,84 & $\begin{array}{l}5,44- \\
11,10\end{array}$ & 16,30 & $\begin{array}{l}13,02- \\
20,75\end{array}$ \\
\hline $\begin{array}{l}\text { Femenino ( } \mathrm{n} \\
=149)\end{array}$ & 98,70 & $\begin{array}{l}94,93- \\
99,94\end{array}$ & 34,90 & $\begin{array}{l}27,7- \\
42,85\end{array}$ & 88,50 & $\begin{array}{l}82,40- \\
92,84\end{array}$ & 18,79 & $\begin{array}{l}13,29- \\
25,87\end{array}$ & 5,36 & $\begin{array}{l}2,58- \\
10,40\end{array}$ & 15,40 & $\begin{array}{l}10,52- \\
22,30\end{array}$ \\
\hline \multicolumn{13}{|l|}{ Edad (años) } \\
\hline $\begin{array}{l}18-29(n= \\
108)\end{array}$ & 97,22 & $\begin{array}{l}91,80- \\
99,40\end{array}$ & 44,40 & $\begin{array}{l}35,42- \\
53,85 \text { * }\end{array}$ & 92,59 & $\begin{array}{l}85,86- \\
96,40\end{array}$ & 20,37 & $\begin{array}{l}13,79- \\
28,99\end{array}$ & 10,19 & $\begin{array}{l}5,62- \\
17,40\end{array}$ & 21,30 & $\begin{array}{l}14,57- \\
29,99 \text { * }\end{array}$ \\
\hline $\begin{array}{l}30-44(n= \\
228)\end{array}$ & 97,81 & $\begin{array}{l}94,82- \\
99,21\end{array}$ & 39,30 & $\begin{array}{l}33,20- \\
45,76\end{array}$ & 92,56 & $\begin{array}{l}88,18- \\
95,44\end{array}$ & 20,18 & $\begin{array}{l}13,66- \\
28,74\end{array}$ & 7,00 & $\begin{array}{l}4,29- \\
11,10\end{array}$ & 19,30 & $\begin{array}{l}14,68- \\
24,94 \text { * }\end{array}$ \\
\hline $\begin{array}{l}45-64(n= \\
170)\end{array}$ & 97,06 & $93,1-98,93$ & 51,10 & $\begin{array}{l}43,72- \\
58,58\end{array}$ & 92,35 & $\begin{array}{l}87,25- \\
95,58\end{array}$ & 20,81 & $\begin{array}{l}15,39- \\
27,49\end{array}$ & 5,00 & $\begin{array}{l}2,67- \\
9,88\end{array}$ & 9,00 & $\begin{array}{l}5,78- \\
14,83\end{array}$ \\
\hline \multicolumn{13}{|l|}{ Estrato } \\
\hline Bajo $(n=271)$ & 97,05 & $\begin{array}{l}94,10- \\
98,60\end{array}$ & 41,30 & $\begin{array}{l}35,63- \\
47,27\end{array}$ & 91,51 & $\begin{array}{l}87,54- \\
94,33\end{array}$ & 17,71 & $\begin{array}{l}13,61- \\
22,72\end{array}$ & 4,79 & $\begin{array}{l}2,75- \\
8,10 \text { * }\end{array}$ & 14,02 & $\begin{array}{l}10,36- \\
18,69\end{array}$ \\
\hline Alto $(n=235)$ & 97,87 & $\begin{array}{l}94,90- \\
99,20\end{array}$ & 48,00 & $\begin{array}{l}41,78- \\
54,45\end{array}$ & 94,04 & $\begin{array}{l}90,17- \\
96,50\end{array}$ & 23,83 & $\begin{array}{l}18,82- \\
29,69\end{array}$ & 9,78 & $\begin{array}{l}6,55- \\
14,31\end{array}$ & 19,15 & $\begin{array}{l}14,61- \\
24,68\end{array}$ \\
\hline \multicolumn{13}{|l|}{ Nivel educativo } \\
\hline $\begin{array}{l}\text { Básico, medio } \\
\text { y técnico }(n= \\
134)\end{array}$ & 96,30 & $\begin{array}{l}91,40- \\
98,64\end{array}$ & 57,40 & $\begin{array}{l}49,00- \\
65,51\end{array}$ & 94,07 & $\begin{array}{l}88,57- \\
97,14\end{array}$ & 18,52 & $\begin{array}{l}12,81- \\
25,97\end{array}$ & 7,00 & $\begin{array}{l}3,38- \\
12,34\end{array}$ & 14,80 & $\begin{array}{l}9,72- \\
21,80\end{array}$ \\
\hline $\begin{array}{l}\text { Pregrado y } \\
\text { posgrado }(n= \\
372)\end{array}$ & 97,85 & $\begin{array}{l}95,73- \\
98,98\end{array}$ & 55,10 & $\begin{array}{l}50,03- \\
60,08\end{array}$ & 92,18 & $\begin{array}{l}88,97- \\
94,54\end{array}$ & 21,29 & $\begin{array}{l}17,42- \\
25,75\end{array}$ & 7,00 & $\begin{array}{l}5,01- \\
10,42\end{array}$ & 16,80 & $\begin{array}{l}13,49- \\
21,15\end{array}$ \\
\hline \multicolumn{13}{|l|}{$\begin{array}{l}\text { Ocupación los } \\
\text { últimos } 30 \text { días }\end{array}$} \\
\hline $\begin{array}{l}\text { Trabajar y/o } \\
\text { estudiar }(n= \\
459)\end{array}$ & 97,38 & $\begin{array}{l}95,43- \\
98,55\end{array}$ & 43,70 & $\begin{array}{l}39,32- \\
48,36\end{array}$ & 93,94 & $\begin{array}{l}88,32- \\
97,07\end{array}$ & 20,52 & $\begin{array}{l}17,07- \\
24,47\end{array}$ & 72,00 & $\begin{array}{l}5,15- \\
9,97\end{array}$ & 50,66 & $\begin{array}{l}46,09- \\
55,21\end{array}$ \\
\hline $\begin{array}{l}\text { Buscar trabajo } \\
(n=11)\end{array}$ & 100,00 & $\begin{array}{l}69,98- \\
100,00\end{array}$ & 54,50 & $\begin{array}{l}27,99- \\
78,75\end{array}$ & 91,8 & $\begin{array}{l}86,30- \\
94,80\end{array}$ & 18,18 & $\begin{array}{l}3,98- \\
48,85\end{array}$ & 90,91 & $\begin{array}{l}0,00- \\
39,90\end{array}$ & 54,55 & $\begin{array}{l}27,99- \\
78,75\end{array}$ \\
\hline $\begin{array}{l}\text { Pensionado } \\
\text { o jubilado ( } \mathrm{n} \\
=14 \text { ) }\end{array}$ & 93,33 & $\begin{array}{l}68,16- \\
100,00\end{array}$ & 50,00 & $\begin{array}{l}30,72- \\
69,28\end{array}$ & 100,00 & $\begin{array}{l}38,26- \\
100,00\end{array}$ & 14,29 & $\begin{array}{l}2,76- \\
41,19\end{array}$ & 0,00 & $\begin{array}{l}0,00- \\
25,15\end{array}$ & 36,36 & $\begin{array}{l}19,64- \\
57,14\end{array}$ \\
\hline $\begin{array}{l}\text { Tareas del } \\
\text { hogar }(n=21)\end{array}$ & 95,45 & $\begin{array}{l}76,49- \\
100,00\end{array}$ & 50,00 & $\begin{array}{l}26,80- \\
73,20\end{array}$ & 92,82 & $\begin{array}{l}88,22- \\
95,77\end{array}$ & 27,270 & $\begin{array}{l}12,88- \\
48,43\end{array}$ & 45,00 & $\begin{array}{l}0,00- \\
23,51\end{array}$ & 28,57 & $\begin{array}{l}11,34- \\
55,03\end{array}$ \\
\hline
\end{tabular}

IC95\%: intervalo al 95\% de confianza.

* Nivel de significancia valor de $p=0,05$ 
Estado de preparación según el Physical Activity Readiness Questionnaire for Everyone (Par-Q+) y análisis bivariado, según variables sociodemográficas de los inscritos a una carrera de fondo. Bogotá, Colombia, 2014.

\begin{tabular}{|c|c|c|c|}
\hline Score Par-Q+ & n & $\%$ & $\mathrm{IC} 95 \%$ \\
\hline Sí (con una condición afirmativa) & 100 & 19,76 & $16,53-23,45$ \\
\hline \multicolumn{4}{|l|}{ Par-Q+ } \\
\hline D01. Condición cardiovascular o presión arterial alta & 18 & 3,56 & $2,18-5,45$ \\
\hline D02. Dolor en el pecho en reposo o en actividad física & 12 & 2,37 & $1,29-3,99$ \\
\hline D03. Pérdida del equilibrio, mareo, o pérdida de conciencia & 8 & 1,58 & $0,73-2,98$ \\
\hline D04. Diagnóstico de condición crónica diferente de cardiovascular e hipertensión & 27 & 5,34 & $3,61-7,56$ \\
\hline D05. Consume medicamento por condición crónica & 39 & 7,71 & $5,61-10,28$ \\
\hline D06. Problemas articulares & 51 & 10,08 & $7,67-12,94$ \\
\hline D07. Doctor recomienda actividad física supervisada & 29 & 5,73 & $3,94-8,02$ \\
\hline Análisis bivariado Par-Q+ vs. variables sociodemográficas & Sí (n) & $\%$ & IC95\% \\
\hline \multicolumn{4}{|l|}{ Categorías Par-Q+ } \\
\hline Hombres & 69 & 19,33 & $15,55-23,75$ \\
\hline Mujeres & 31 & 20,81 & $15,02-28,06$ \\
\hline $18-29$ & 19 & 17,59 & $11,48-25,93$ \\
\hline $30-44$ & 36 & 15,79 & $11,60-21,12$ \\
\hline $45-64$ & 45 & 26,47 & $20,39-33,59$ \\
\hline Estrato 1-2-3 & 50 & 18,45 & $14,26-23,52$ \\
\hline Estrato 4-5-6 & 50 & 21,28 & $16,51-26,97$ \\
\hline Básico, Medio, Técnico, otro & 23 & 17,04 & $11,57-24,33$ \\
\hline Pregrado-Posgrado & 77 & 20,75 & $16,93-25,18$ \\
\hline Trabajar y/o estudiar & 82 & 17,90 & $14,65-21,69$ * \\
\hline Buscar trabajo & 3 & 27,27 & $9,20-57,10$ \\
\hline Tareas del hogar & 7 & 31,82 & $16,19-52,85$ \\
\hline Pensionado o jubilado & 7 & 50,00 & $26,80-73,20$ \\
\hline \multicolumn{4}{|l|}{ Cumplimiento de actividad física } \\
\hline No cumplen & 4 & 30,77 & $12,35-57,96$ \\
\hline Sí cumplen & 96 & 19,47 & $16,21-23,21$ \\
\hline \multicolumn{4}{|l|}{ Consumo de frutas y verduras } \\
\hline Bajo y muy bajo & 91 & 19,65 & $16,28-23,53$ \\
\hline Óptimo y sub-óptimo & 7 & 18,92 & $9,17-34,51$ \\
\hline
\end{tabular}

IC95\%: intervalo al 95\% de confianza.

* Nivel de significancia valor de $p=0,05$.

comportamientos relacionados con la dieta saludable, el consumo de tabaco y de alcohol. Se requiere una perspectiva integral de las condiciones previas de participación, que le conviertan en una oportunidad para promover comportamientos saludables 52,53.

Abordar la premisa de que los corredores recreativos son "activos y saludables" requiere ampliar el estudio desde una perspectiva de valoración de los factores riesgo ${ }^{54}$. Los resultados obedecerían a una fase previa de carrera que no asegura que sean comportamientos permanentes.
Por tal razón, surgen preguntas como: ¿qué implicaciones existen en la relación alcohol y actividad física?, ¿qué efectos frente a las enfermedades crónicas tiene el bajo consumo de frutas y verduras en los corredores aficionados o recreativos?

Este estudio constituye evidencia científica de los factores de riesgo del comportamiento, relacionados con el estilo de vida en la población de corredores aficionados. Es un punto de partida, para el estudio del movimiento del atletismo de calle como una estrategia que promueve hábitos y comportamientos saludables, y por ende aporta 
en la prevención de enfermedades no transmisibles. Se hace necesario proyectar otros estudios que incluyan mediciones bioquímicas (colesterol HDL, LDL glicemia, presión arterial y medición del consumo de oxígeno) como indicadores de salud, así como la historia familiar para puntualizar en la estratificación del riesgo desde el punto de vista cardiovascular.

Aunque se caracteriza como "saludables" a los participantes desde el cumplimiento de los niveles de actividad física, existe un grupo a considerar desde el reporte del Par-Q+ que necesita tener unos mecanismos prevención y seguimiento; para la seguridad en la participación. Shepard 55 sugiere utilizar el PARmed-X 24 para afinar y lograr estratificar el riesgo y así potenciar la oportunidad de participación en la actividad física.

\section{Colaboradores}

M. P. Ramírez-Góngora colaboró en la concepción e implementación del proyecto de investigación, análisis e interpretación de los datos, redacción del artículo y revisión crítica relevante del contenido intelectual. F. E. Prieto-Alvarado participó en la asesoría del proyecto investigación, análisis e interpretación de los datos, redacción del artículo y revisión crítica relevante del contenido intelectual. Los dos autores son responsables de todos los aspectos del trabajo, garantizando la exactitud e integridad.
El instrumento utilizado en el estudio es una herramienta que puede llegar a convertirse en un protocolo de conocimiento y valoración del riesgo potencial de los inscritos a carreras atléticas, siendo un valor agregado para los participantes quienes evidenciarán de primera mano el interés de los organizadores por ofrecer eventos atléticos seguros.

Entre las limitaciones que tiene el estudio encontramos la posible sobrevaloración de los niveles de actividad física, teniendo en cuenta que, los participantes se encuentran en el previo de la carrera. Por otro lado, no ha sido posible obtener las estadísticas de eventos o emergencias médicas durante y post carrera derivadas de la participación en las mismas.

\section{Agradecimientos}

A la Organización Correcaminos de Colombia, Organizadores Media Maratón de Bogotá D.C., Cardio Street BP Ltda., Instituto Distrital de Recreación y Deporte I.D.R.D en cabeza de Rocío Gámez del Programa Muévete Bogotá, Secretaría Distrital de Salud de Bogotá, Liga de Atletismo de Bogotá, Grupo CEMA Universidad del Rosario, Jhon Duperly PhD. A los veinte encuestadores con perfil licenciados en Educación Física y áreas afines a la salud. 


\section{Referencias}

1. Kim J, Malhotra R, Chiampas G, D'Hemecourt P, Troyanos C, Cianca J, et al. Cardiac arrest long distance running race. N Engl J Med 2012; 366:130-40.

2. Siegel A. Pheidippides redux: reducing risk for acute cardiac events during marathon running. Am J Med 2012; 125:630-5.

3. Glover D, Maron B. Profile of preparticipation cardiovascular screening for high school athletes. JAMA 1998; 279:1817-9.

4. Leischik R, Littwitz H, Dworrak B, Spelsberg N, Tiroch K. Cardiovascular screening for recreational, leisure, vigorous and competitive sport activities over 35 years. Dtsch Med Wochenschr 2014; 139:2188-94.

5. Liga de Atletismo de Bogotá. Calendario atlético 2014. http://www.ligatletismobogota.com/ima ges/pdfs/calendario\%20atletico\%202014.pdf (accedido el 12/Abr/2014).

6. Alcaldía Mayor de Bogotá. Proyecto de Acuerdo $\mathrm{n}^{\circ}$ 186, de 2011. Por medio del cual se declara de interés cultural la media maratón de Bogota como evento deportivo y recreativo y se dictan otras disposiciones. http://www.alcaldiabogota.gov.co/ sisjur/normas/Norma1.jsp?i=43307 (accedido el 23/Ene/2014).

7. Maron BJ, Epstein E, Roberts WC. Causes of sudden death in competitive athletes. J Am Coll Cardiol 1986; 7:204-14.

8. Maron B. Risk profiles and cardiovascular preparticipation screening of competititve athletes. Cardiol Clin 1997; 15:473-83.

9. Corrado D, Basso C, Schiavon M, Pellicia A, Thiene G. Cardiovascular pre-participation screening of young competitive athletes. Eur Heart J 2005; 26:516-24.

10. Calderón JC, Fernández AZ, Jesús AIM. Ateroesclerosis, estrés oxidativo y actividad física. Invest Clín 2008; 49:397-410.

11. Ramírez-Vélez R, Ramírez JH, Aguilar C. Cambios em la función vascular de hombres saludables después de ejercicio físico prolongado y vigoroso. Rev Colomb Cardiol 2010; 17:203-6.

12. James J, Merghani A, Sharma S. Sudden death in marathon runners. Cardio Electrophysiol Clin 2013; 5:43-51.

13. Albert C, Murray A, Mittleman A, Chae C, Lee I-M, Hennekents C. Triggering of sudden death from cardiac causes by vigorous exertion. New Eng J Med 2014; 343:1355-60.

14. Schmied CM. Improvement of cardiac screening in amateur athletes. J Electrocardiol 2015; 48:351-5.

15. Haeusler KHJ, Kunze C, Krüll M, Brechtel L, Lock J. Rate of cardiac arrhythmias and silent brain lesions in experienced marathon runners: rationale, design and baseline data of the Berlin Beat of Running Study. BMC Cardiovasc Disord 2012; 12:69.

16. Bas Kluitenberg M, Diercks R, Hartgens F, Verhage E, Smits D, Buist I. The NLstart2run Study: health effects of a running promotion program in novice runners, design of a prospective cohort study. BMC Public Health 2013; 13:685.

17. Sheppard M. The fittest person in the morgue? Histopatology 2012; 60:381-96.
18. Möhlenkamp S, Schmermund A, Kröger K, Kerkhoff G, Bröcker-Preuss M, Adams V, et al. Coronary atherosclerosis and cardiovascular risk in masters male in marathon runners. Herz 2006; 31:575-85.

19. Instituto Colombiano de Bienestar Familiar. Encuesta Nacional de la Situación Nutricional en Colombia 2010. Bogotá: Instituto Colombiano de Bienestar Familiar; 2011.

20. Craigh C, Marshall A, Sjötröm M, Bauman A, Booth M, Ainsworth B, et al. International Physical Activity Questionnaire: 12-country reliability and validity. Med Sci Sports 2003; 35:1381-95.

21. Hallal PC, Gómez LF, Parra DC, Lobelo F, Mosquera J, Florindo AA, et al. Lecciones aprendidas después de 10 años del uso de IPAQ en Brasil y Colombia. J Phys Act Health 2010; 7 Suppl 2:S259-64.

22. Brendin SS, Gledhill N, Jamnik VK, Warburton DE. Par-Q+ and ePARmed-X+: new risk stratification and physical activity clearance strategy for physicians and patients alike. Can Fam Physician 2013; 19:273-7.

23. Warburton DE, Shannon S, Brendin SS. The new Par-Q+ and ePARmed-X+: latest advancements in exercise clearance, risk stratification, and prescription. http://forms.acsm.org/Summit2013/ pdfs $/ 50 \% 20$ Warburton\%20Bredin.pdf (accedido el 20/Oct/2014)

24. Warburton DE, Jamnik VK, Bredin SS, Gledhill N. The 2014 Physical Activity Readiness Questionnaire for Everyone (Par-Q+) and electronic Physical Activity Readiness Medical Examination (ePARmed-X+). Health \& Fitness Journal of Canada 2014; 7:80-3.

25. World Health Organization. Chronic diseases and health promotion: STEPS conceptual framework. http://www.who.int/chp/steps/framework/en/ (accedido el 20/Oct/2013).

26. Thompson PD, Franklin BA, Balady GJ, Blair SN, Corrado D, Estes 3rd NA, et al. Exercise and acute cardiovascular events placing the risks into perspective: a scientific statement from the American Heart Association Council on Nutrition, Physical Activity, and Metabolism and the Council on Clinical Cardiology. Circulation 2007; 115:2358-68.

27. Haskell W, Lee IM, Pate RR, Powell KE, Blair SN, Franklin BA, et al. Physical activity and public health: updated recommendation for adults from the American College of Sports Medicine and the American Heart Association. Med Sci Sports Exerc 2007; 39:1423-34.

28. Warburton DE, Brendin SS, Jamnik VK, Gledhill N. Validation of the Par-Q+ and ePARmed.X+. Health \& Fitness Journal of Canadá 2011; 4:38-46.

29. World Health Organization. Global Status Report on noncommunicable diseases. http://www.who. $\mathrm{int} / \mathrm{nmh} /$ publications/ncd-status-report-2014/ es/ (accedido el Ago/2014).

30. Organización Mundial de la Salud. Informe sobre la situación mundial de las enfermedadas no transmisibles 2014. Geneva: Organización Mundial de la Salud; 2014. 
31. Organización Mundial de la Salud; Organización de las Naciones Unidas para la Agricultura y la Alimentación. Un marco para la promoción de frutas y verduras. Geneva: Organización Mundial de la Salud; 2005.

32. Pate RR, Pratt M, Blair SN, Haskell WL, Macera CA, Bouchard C, et al. Physical activity and public health. A recommendation from the Centers for Disease Control and Prevention and the American College of Sports Medicine. JAMA 1995; 273:402-7.

33. Berry G, Armitage P. Mid-P confidence intervals: a brief review. Statisticians 1995; 44:417-23.

34. Donado C, Duperly J, Lobelo F, Ramírez A, Montoya E. Prevalence of risk factors for recreational race-associated cardiovascular events among runners in Bogota City. Med Sci Sports Exerc 2011; 43 Suppl 1:345-6.

35. Basso C, Schiavon M, Pellicia A, Thiene G. Cardiovascular pre-participation screening of young competitive athletes. Eur Heart J 2005; 26:516-24.

36. Cohen SI, Ellis ER. Death and near death from cardiac arrest during the Boston Marathon. Pacing Clin Electrophysiol 2012; 35:241-4.

37. Priori SG, Aliot E, Blomstrom-Lundqvist C, Bossaert L, Breithardt G, Brugada P, et al. Task force on sudden cardiac death of the European Society of Cardiology. Eur Heart J 2001; 22:1374-450.

38. Warburton DE, Gledhill N, Jamnik VK, Bredin SS, McKenzie DC, Stone J, et al. Evidence-based risk assessment and recommendations for physical activity clearance: Consensus Document 2011. Appl Physiol Nutr Metab 2011; 36 Suppl 1:S266-98.

39. Shephard RJ. Can we identify those for whom exercise is hazardous? Sports Med 1984; 1:75-88.

40. Zwerver J, Bessem B, Buist I, Diercks R. The value of preventive advice and examination focusing on cardiovascular events and injury for novice runners. Ned Tijdschr Geneeskd 2008; 152:1825-30.

41. Schmied C, Borjesson M. Sudden cardiac death in athletes. J Intern Med 2014; 275:93-103.

42. Observatorio de Salud Pública de Santander, Secretaría de Salud de Santander. Factores de riesgo para enfermedades crónicas en Santander, método STEPwise. Bucaramanga: Universidad Industrial de Santander; 2011.

43. Ezzati M, Riboli E. Behavioral and dietary risk factors for noncommunicable diseases. N Engl J Med 2013; 369:954-64.
44. Jacoby E, Keller I. La promoción promoción del consumo de frutas y verduras en América Latina: buena oportunidad de acción intersectorial por una alimentación saludable. Rev Chil Nutr 2006; 33 Suppl 1:226-31.

45. World Health Organization. Global Burden disease study country report, 2010. http://www.health metricsandevaluation.org (accedido el Sep/2013).

46. Stolarz-Skrzypek K, Staessen JA. Reducing salt intake for prevention of cardiovascular disease: times are changing. Adv Chronic Kidney Dis 2015; 22:108-15.

47. Azak A, Huddam B, Gonen N, Yilmaz SR, Kocak G, Duranay M. Salt intake is associated with inflammation in chronic heart failure. Int Cardiovasc Res J 2014; 8:89-93.

48. Gómez Gutiérrez LF, Lucumí Cuesta DI, Girón Vargas SL, Espinosa García G. Conglomeración de factores de riesgo de comportamiento asociados a enfermedades crónicas en adultos jóvenes de dos localidades de Bogotá, Colombia: importancia de las diferencias de género. Rev Esp Salud Pública 2004; 78:493-504.

49. World Health Organization. WHO report on the global tobacco epidemic: executive summary. Geneva: World Health Organization; 2013.

50. Ortega R, Aguilar E. Influencia de la carrera a pie sobre el hábito tabáquico. Aten Prim (Barc.) 2006; 37:478-83.

51. Giraldo G, Sierra-Heredia C, Giraldo F, Valdelamar F, Ramírez A, Sansores S. Impacto del tabaquismo en el rendimiento físico. Rev Inst Nac Enfermedades Respir 2001; 14:215-9.

52. Poortinga $W$. The prevalence and clustering of four major lifestyle risk factors in an English adult population. Prev Med 2007; 44:124-8.

53. Poortinga W. Associations of physical activity with smoking and alcohol consumption: a sport or occupation effect? Prev Med 2007; 45:66-70.

54. Organización Mundial de la Salud. 10 datos sobre las enfermedades no transmisibles. http://www. who.int/features/factfiles/noncommunicable_di seases/es/ (accedido el 03/Mar/2014).

55. Shepard R. Qualified exercise and fitness as professionals and exercise prescription: evolution of the Par-Q and Canadian Aerobic Fitness Test. J Phys Act Health 2015; 12:454-61. 


\section{Abstract}

Participation in amateur street marathons has become increasingly popular and requires prior individual health risk assessment. The objective was to identify risk factors and readiness in registered runners. This was a cross-sectional study in a random sample $(n=510)$ of registered amateur runners 18-64 years of age, using a digital survey with IPAQ, Par-Q+, and STEPwise, with an expected physical inactivity rate of $35 \%( \pm 5 \%)$. The study explored physical activity, (binge) alcohol consumption, fruit, vegetable, and salt intake, smoking, and readiness. Self-reported rates were: $97.4 \%$ recommended level of physical activity, 2.4\% optimal fruit and vegetable intake, $3.7 \%$ smoking, and $44.1 \%$ binge drinking. $19.8 \%$ were Par-Q+ positive and $5.7 \%$ practiced supervised exercise. The analysis showed differences by age, sex, and socioeconomic status. Recreational runners followed the recommended levels of physical activity but did not score well on other risk factors. Prior evaluation of lifestyle-related risk factors and readiness provides a safer athletic strategy.

Running; Sudden Death; Athletes; Chronic Disease; Risk Factors

\section{Resumo}

A participação em corridas de rua tem aumentado; isto requer uma detecção de riscos prévios ao esforço físico. O objetivo foi identificar os fatores de risco de comportamento e de readiness nos inscritos em uma corrida atlética. Estudo transversal em aficionados de 18 a 64 anos. Foi aplicado um questionário digital com módulos do IPAQ, Par-Q+e STEPwise. Uma amostra aleatória sistemática, $n=510$, para uma inatividade física estimada em $35 \%$ ( $\pm 5 \%$ ). Avaliou-se o nivel de atividade física, o consumo perigoso de álcool, fruta, verdura tabaco, sal e readiness. A realização de atividade física foi de 97,4\%; 2,4\% consomem niveis ideais de frutas ou verduras (diferenças de idade, sexo e estrato); 3,7\% são fumantes; e 44,1\% consomem álcool. Dezenove vírgula oito por cento relataram Par-Q+ positivo e 5,7\% requerem exercício supervisionado. Existem diferenças de trabalho e estudo. Os corredores recreacionais atendem aos níveis de atividade física, mas não de outros fatores. Uma estratégia de atletismo seguro é avaliar os fatores de risco relacionados ao estilo de vida e readiness.

Corrida; Morte Súbita; Atletas; Doença Crônica Fatores de Risco
Recibido el 26/Abr/2015

Versión final presentada el 10/Jul/2015

Aprobado el 27/Ago/2015 